\title{
Research on Optimization of Risk Allocation in Enthusiasm Mechanism of Enterprise: Based on a Dynamic Game Model
}

\author{
Shang Hua ${ }^{1,2}$ and Hui Shan-cheng ${ }^{3}$ \\ ${ }^{1,2}$ First Author \& Correspondence Author Faculty of Management and \\ Economics, \\ Dalian University of Technology, Dalian China 116024, shanghua@dlut.edu.cn \\ ${ }^{3}$ Faculty of Management and Economics, Dalian University of Technology \\ Dalian China 116024, huishch08@163.com
}

\begin{abstract}
Risk allocation is an important path to study enthusiasm mechanism. Based on the analysis of a dynamic game model, this paper analyzes the equilibrium game strategy which under the different initial conditions. And then, we analyze the different proportion of risk allocation is how to impact on performance and lead the enthusiasm mechanism to fail. Based on the equilibrium game strategy under the different initial conditions, the evolution of risk allocation and the failure in motivation mechanism has been explored. As a consequence, the benefits and costs when employees work hard and the rewards and punishments in motivation mechanism will influence the outcome of the dynamic game directly. The optimal proportion of risk allocation in dynamic game is helpful to realize the optimization of motivation mechanism, and then we put forward the optimal proportion of risk allocation conditions should be matched.
\end{abstract}

Keywords: Enthusiasm mechanism, Risk allocation, Dynamic game model, Optimization, Optimal ratio

\section{Introduction}

Human resources became strategic resources in modern economic development when they replaced traditional material resources. In enterprise management, enterprise tends to stimulate employee productivity by using the by using the motivation mechanism in order to improve employee performance, while at the same time encouraging employees to achieve strategic goals of the enterprises ${ }^{[1]}$. The motivation mechanism is a core issue, which is focused on by enterprises and employees. The purpose of the motivation mechanism is not only to maximize the benefits of the principal but to maximize the benefits of the agent.

However, the asymmetric information and the inconsonant benefits between the principal and the agent may compromise an enterprises' motivation mechanism. The risk of the motivation mechanism failing will lead to a decline in employee performance and to the enterprise's performance, a challenge to the enterprise's culture, and even an increase in employee turnover. Furthermore, it leads to a decreased economic efficiency. The enterprise and the employee should allocate the risk reasonably and impartially according to the principle of risk allocation, i.e., the ratio of risk allocation should be focused ${ }^{[2]}$. If the enterprise allocates much more risk, insufficient competition can easily

Projected Supported: National foundational of social science (12BGL067); Fund for Educational and Teaching Reform of DUT(MS2014007); Fund for Graduate Teaching Reform and Research of DUT(JG2014018); Fund for Graduate Teaching Reform and Research of DUT(JG2014002) 
to make employees lose sight of goals and their working enthusiasm, eventually leading to the failure motivation mechanism. However, if the ratio of risk allocation of employee is too high, excessive pressure can easily to make employees lose a seriousness of purpose within work and thus cause them to deviate from the goal of enterprises. Eventually, the motivation mechanism fails. A reasonable ratio of risk allocation between the enterprise and its employees is the key to the success of the enterprise's motivation mechanism. Therefore, through an empirical analysis of risk allocation impact on enterprise performance and employee performance, this paper addresses (1) the reason why enterprise motivation mechanism fails, (2) the process of achieving an optimal ratio of risk allocation between enterprises and employees thus improving both employee performance the optimization of an enterprise's motivation mechanism.

\section{Theoretical Background}

\subsection{Principal-Agent Theory}

The principal-agent theory describes the contractual relationship between the principal and the agent. This contractual relationship widely exists in enterprises. The operators of an enterprise authorize agents engaged in management activities through a contract ${ }^{[3]}$. A key assumption in the principal-agent theory is the asymmetry of information and the inconsistent benefit between the principal and the agent, which results in a high agent cost ${ }^{[4]}$ and an unpredictable risk in the enterprises' motivation mechanism ${ }^{[5]}$. Meanwhile, the "participation constraint" and "incentive compatibility" models also emphasize that an agent must allocate part of risk ${ }^{[6]}$. Mirrlees emphasized that the principal and the agent should seek the optimal motivation mechanism within the framework of benefit sharing and the risk allocation contract ${ }^{[7]}$. Moreover, Terpstra and Rozell demonstrated that there is a positive correlation between a reasonable motivation mechanism and enterprise performance and employee performance ${ }^{[8]}$.

Previous research has focused on the contractual relationship between an enterprise and an employee, the risk allocation between an enterprise and an employee, and the optimization of the motivation mechanism. However, from the principal-agent relationship between enterprise and employee, there is no theoretical and empirical proof to analyze the optimization of motivation mechanism, and the previous research has not analyzed the main reason behind the failure of the motivation mechanism. Therefore, the optimization of the motivation mechanism should be discussed in terms of a reasonable ratio of risk allocation which helps determine the success of the motivation mechanism.

\subsection{Research on Risk Allocation}

Research on risk allocation is centered on the project management. Akintoye, Taylor and Fitzgerald summarized the phenomenon of "existing risk" during project implementation in their research ${ }^{[9]}$. Qi, Ke and Wang put forward the concept of "key risk" during project management through a case study ${ }^{[10]}$. According to Law and Chow, risks, which are influenced by the processes of the project, were summarized and collated ${ }^{[11]}$. Li, in his study, described the influenced of both the principal's and the agent's risk preference on risk allocation, in addition to demonstrating the necessity of risk allocation while simultaneously asserting the associated rule ${ }^{[12]}$. Song analyzed risk allocation based on the risk allocation relationship between a principal and an agent ${ }^{[13]}$. Shen and his team illustrated how risk allocation affected the project performance and they emphasized that a reasonable ratio of risk allocation is important to a project's performance ${ }^{[14]}$.

During project management, research on risk allocation has become mature. An enterprise will face the same risks when attempting to implement a motivation mechanism. However, the existing research on risk allocation in motivation mechanism is sparse. Based on the principal-agent model, Duan and Yuan stressed that asymmetry in 
information access and the differences that arise during risk identification are the main reason leading to breakdown of the principal-agent contract broken ${ }^{[15]}$. In game theory, incomplete contract theory predicts how the rules of risk allocation affected an agent's moral behavior ${ }^{[16]}$. Game theory suggests that a reasonable ratio of risk allocation could make up for the contract defects ${ }^{[17]}$. Until now, there are few scholars have analyzed the ratio of risk allocation between principal and agent, few studies have explored the optimal ratio of risk allocation between principal and agent. Furthermore, there is no theoretical and empirical proof to determine the exact reason for when the motivation mechanism fails.

According to the studies referenced above, this paper uses the risk allocation methods from the project management for reference, regarding different types of risk as a risk variable. Then, based on principal-agent theory, we discuss the influence of different ratios of risk allocation to operating motivation mechanisms as well as to enterprise and employee performance from the perspective of both principal and agent. Essentially, this paper suggests that an unreasonable ratio of risk allocation leads to the failure of an operating motivation mechanism and goes on to discuss the optimal ratio of risk allocation between principal and agent to avoid risks and achieve an optimally functioning motivation mechanism.

\section{Analysis of Risk Allocation}

\subsection{A game Model and Hypothesizes}

Under the condition of symmetrical information, for agents, the optimal choice is for them to maximizing their benefit through hard work. However, for principals, the optimal choice is not to motivate employees. Therefore, under the condition of symmetrical information, Nash equilibrium between enterprises and employees is (not motivate, work hard). In this instance, the motivation mechanism does not exist. However, an asymmetry of information between principal and agent is common. Because of the asymmetry of information, enterprises, which are bounded rationality, cannot supervise employees' behavior completely. Enterprises motivate employees to work hard by using some sort of motivation mechanism. However, the potential risk in any motivation mechanism is determined by the uncertainty of the employees' behaviors, which means that enterprises and employees ought to allocate risk. And a reasonable ratio of risk allocation is a key to avoid any failure of the motivation mechanism.

Referred to the literature on the benefits of enterprises and employee under different strategies ${ }^{[18-20]}$, and in this paper we do not change the nature of the research. The hypothesis model has been explained. The enterprise, as an organization hiring labor, is a broad concept, and it refers to principal. The employee refers to agent. Under this condition, we assume that the enterprise provides $\mathrm{W}$ for employees to motivate them work hard. If employees do not work hard, the punishment is P. The risk cost of the enterprise is $\mathrm{C}$, and the profit is R. The benefit of employees is $U$ when they work hard. Employees will get negative utility due to work hard, and the cost is Q.

In the process of a dynamic game of motivation mechanism, we assume that the probability of the enterprise provides motivation mechanism isx, and the probability of the enterprise does not provide motivation mechanism is $1-\mathrm{x}$. Then, the probability of employees work hard is $\mathrm{y}$, and the probability of employees do not work hard is $1-\mathrm{y}$. The benefit is $R_{1 A}$ when the enterprise provides motivation mechanism, and the benefit is $R_{2 A}$ when the enterprise does not provide motivation mechanism. The expected benefit is $\overline{R_{A}}$. The following formula can be computed:

$$
\begin{gathered}
\mathrm{R}_{1 \mathrm{~A}}=(-\mathrm{C}-\mathrm{W})+(1-\mathrm{y})(-\mathrm{M}-\mathrm{C}+\mathrm{P}) \\
=\mathrm{y}(\mathrm{M}-\mathrm{W}-\mathrm{P})+\mathrm{P}-\mathrm{M}-\mathrm{C} \\
\mathrm{R}_{2 \mathrm{~A}}=\mathrm{yM}-\mathrm{M} \\
\overline{\mathrm{R}_{\mathrm{A}}}=\mathrm{xR}_{1 \mathrm{~A}}+(1-\mathrm{x}) \mathrm{R}_{2 \mathrm{~A}}=-\mathrm{xy}(\mathrm{W}+\mathrm{P})+\mathrm{x}(\mathrm{P}-\mathrm{C})+(\mathrm{y}-1) \mathrm{M}
\end{gathered}
$$


Then, we assume that if employees work hard, they get the incentive compensation is $R_{1 B}$, if employees do not work hard, they get the incentive compensation is $R_{2 B}$, the expected benefit is $\overline{\mathrm{R}_{\mathrm{B}}}$. The following formula can be computed:

$$
\begin{gathered}
\mathrm{R}_{1 \mathrm{~B}}=\mathrm{x}(-\mathrm{Q}+\mathrm{W}+\mathrm{U})+(1-\mathrm{x})(-\mathrm{Q}+\mathrm{U}=\mathrm{xW}+\mathrm{U}-\mathrm{Q}) \\
\mathrm{R}_{2 \mathrm{~B}}=-\mathrm{yP} \\
\overline{\mathrm{R}_{\mathrm{B}}}=\mathrm{yR}_{1 \mathrm{~B}}+(1-\mathrm{y}) \mathrm{R}_{2 \mathrm{~B}}=\mathrm{xy}(\mathrm{W}+\mathrm{P})+\mathrm{y}(\mathrm{U}-\mathrm{Q})-\mathrm{xP}
\end{gathered}
$$

\subsection{The Dynamic Equation of Motivation Mechanism}

We establish a dynamic equation of motivation mechanism as follows:

$$
\mathrm{F}(\mathrm{x})=\frac{\mathrm{dx}}{\mathrm{dt}}=\mathrm{x}\left(\mathrm{R}_{1 \mathrm{~A}}-\overline{\mathrm{R}_{\mathrm{A}}}\right)=\mathrm{x}(\mathrm{x}-1)(\mathrm{yW}+\mathrm{yW}-\mathrm{P}+\mathrm{C})
$$

Let $F(x)=0$, and $y=\frac{P-C}{W+P}, x=0, x=1$ is the steady state of equation.

Derivation of $F(x)$, we get $\frac{d F(x)}{d x}=(2 x-1)(y W+y P-P+C)$

It asks $\frac{d F(x)}{d x}<0$, we must analyze $(P-C)$, two cases as follows:

(1) If $(\mathrm{P}-\mathrm{C})<0$, that is $\frac{\mathrm{P}-\mathrm{C}}{\mathrm{W}+\mathrm{P}}<0, \mathrm{y}>\frac{\mathrm{P}-\mathrm{C}}{\mathrm{W}+\mathrm{P}}, \mathrm{x}=0$ is the steady state of equation.

(2)If $0<(\mathrm{P}-\mathrm{C})<(W+P)$, that is $0<\frac{\mathrm{P}-\mathrm{C}}{\mathrm{W}+\mathrm{P}}<1$, we have two cases.

If $y>\frac{P-C}{W+P},\left.\frac{d F(x)}{d x}\right|_{x=0}<0,\left.\frac{d F(x)}{d x}\right|_{x=1}>0$, and $x=0$ is the steady state of equation.

If $y<\frac{P-C}{W+P},\left.\frac{d F(x)}{d x}\right|_{x=0}>0,\left.\frac{d F(x)}{d x}\right|_{x=1}<0$, and $x=1$ is the steady state of equation.

\subsection{The Dynamic Equation of Employees' Effort}

We establish a dynamic equation of employees' effort as follows:

$$
F(y)=\frac{d y}{d t}=y\left(R_{1 B}-\overline{R_{B}}\right)=y(y-1)(x W+x P+U-Q)
$$

Let $F(x)=0$, and $x=\frac{Q-U}{W+P}, y=0, y=1$ is the steady state of equation. And we must analyze the cases of $(\mathrm{Q}-\mathrm{U})$.

(1) If $(Q-U)<0$, that is $\frac{Q-U}{W+P}<0$, we get $x>\frac{Q-U}{W+P}$, and $y=1$ is the steady state of equation.

(2) If $(\mathrm{Q}-\mathrm{U})>(W+P)$, that is $\frac{\mathrm{Q}-\mathrm{U}}{\mathrm{W}+\mathrm{P}}>1$, we get $\mathrm{x}<\frac{\mathrm{Q}-\mathrm{U}}{\mathrm{W}+\mathrm{P}}$, and $\mathrm{y}=0$ is the steady state of equation.

(3) If $0<(\mathrm{Q}-\mathrm{U})<(W+P)$, that is $0<\frac{\mathrm{Q}-\mathrm{U}}{\mathrm{W}+\mathrm{P}}<1$, two cases as follows:

If $\mathrm{x}>\frac{\mathrm{Q}-\mathrm{U}}{\mathrm{W}+\mathrm{P}},\left.\frac{\mathrm{dF}(\mathrm{y})}{\mathrm{dy}}\right|_{\mathrm{y}=0}>0,\left.\frac{\mathrm{dF}(\mathrm{y})}{\mathrm{dy}}\right|_{\mathrm{y}=1}<0$, and $\mathrm{y}=1$ is the steady state of equation.

If $\mathrm{x}<\frac{\mathrm{Q}-\mathrm{U}}{\mathrm{W}+\mathrm{P}},\left.\frac{\mathrm{dF}(\mathrm{y})}{\mathrm{dy}}\right|_{\mathrm{y}=0}<0,\left.\frac{\mathrm{dF}(\mathrm{y})}{\mathrm{dy}}\right|_{\mathrm{y}=1}>0$, and $\mathrm{y}=0$ is the steady state of equation.

According to the results of the analysis of game models, the trend of dynamic game between enterprises and employees can be seen from Figure 1. According to Figure 1, we can obtain four different equilibrium states.

(1) When the game state region in I, the game model converges to $x=1, y=0$, and the game equilibrium strategy is (motivate, no effort).

(2) When the game state region in II, the game model converges to $x=1, y=1$, and the game equilibrium strategy is (motivate, work hard).

(3) When the game state region in III, the game model converges to $x=0, y=1$, and the game equilibrium strategy is (not motivate, work hard).

(4) When the game state region in IV, the game model converges to $x=0, y=0$, and the game equilibrium strategy is (not motivate, no effort). 
However, four state strategies have not improved to stable strategy.

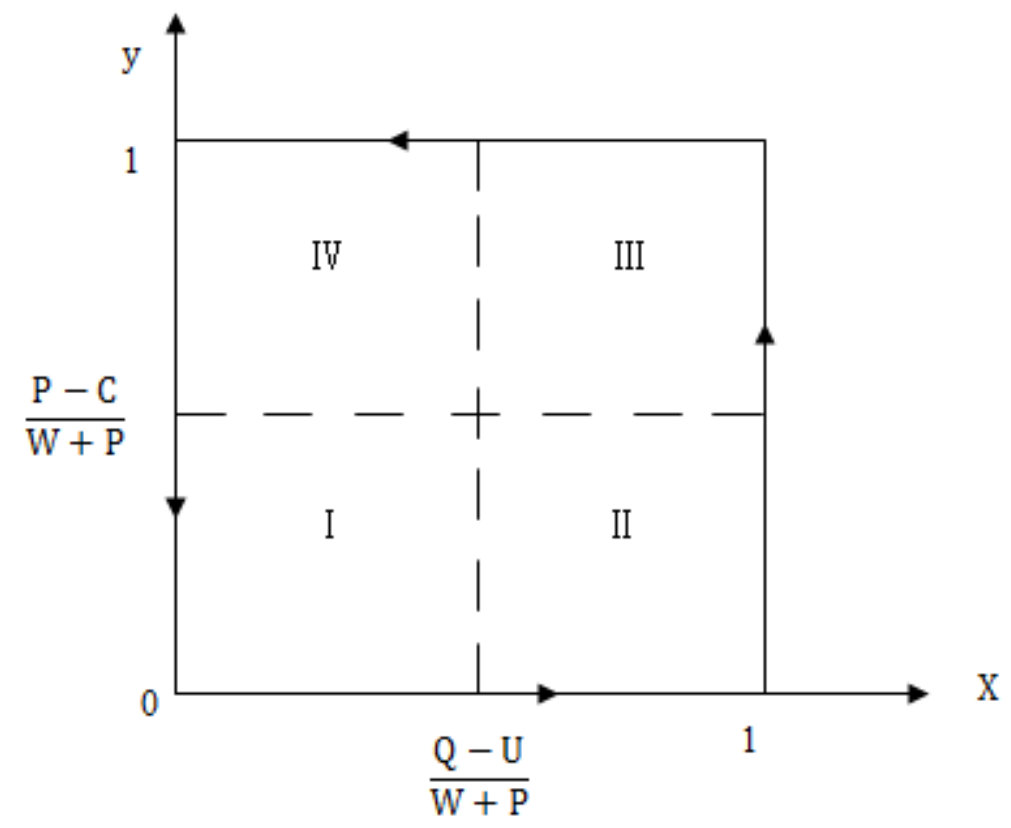

Figure 1. The Evolution of Dynamic Game between Enterprise and

\subsection{Analysis of Risk Allocation}

The first stable strategy indicates the cost of enterprise provides compensation more than the punishment when employees work hard, the enterprise's strategy is not motivation. In addition, if the compensation, which the enterprise provides, is high enough, the probability of employees to work hard will be increased to 1 . However, on the contrary, the probability of employees to work hard will be reduced to 0 . In the motivation mechanism, if the enterprises allocate a larger ratio of risk, they will transfer the risk to employees moderately, and the enthusiasm of employees will increase. However, if the enterprises allocate a lower ratio of risk, they will transfer the risk to employees excessively, and the enthusiasm of employees will be reduced when the ratio of risk allocation of employees is more than the ratio of risk allocation of enterprises. Thus, the enterprises should supervise employees' behavior and increase the punishment for employee's shirking of responsibility and moral behavior in order to motivate employees to allocate risk and reduce the liable to stochastic variation (i.e., randomness).

The second stable strategy shows that it brings more income than cost when employees work hard, and employees' strategy is to work hard. If employees' effort brings more income than cost, the employees' performance will continue to improve. In the motivation mechanism, if the employees allocate a lower ratio of risk, and the enthusiasm of employees increase, and performance will improve. However, on the contrary, if the employees allocate a larger ratio of risk or enterprises transfer much more risk to employees, the enthusiasm of employees will be reduced, and the performance will be reduced. Thus, as a principal, enterprises should adjust the ratio of risk allocation and transfer risk moderately. And it is necessary to guide and help employees when they allocate a larger ratio of risk.

The third stable strategy illustrates that the cost of employee's effort is more than the compensation, the reward and the punishment. The optimal strategy of employee is not to work hard. The motivation mechanism cannot motivate employees to realize the goals when the compensation remains unchanged. In this circumstance, the enterprises motivate employees to work hard by transferring risk to employees and using the competition of culture and market.

However, there is no stable strategy when the game between the enterprise and the 
employee in $(\mathrm{P}-\mathrm{C})>0$ and $0<(\mathrm{Q}-\mathrm{U})<(W+P)$. It indicates that the game between the enterprise and the employee is a dynamic process, and the stable strategy of the game is just phase equilibrium, and exhibits periodic. In motivation mechanism, the ratio of risk allocation between the enterprise and the employee is not stable but to evolve into a state of stable equilibrium.

Because of the asymmetric informational access, enterprises cannot completely supervise employees' behavior, and bounded rational enterprises will motivate employees to work hard and improve performance by increasing incentive compensation. Enterprises create a partially competitive culture while improving incentive compensation. When employees lack the necessary pressure of competition, they may also a lack of goals. When an employee's work behavior deviates from the benefits of enterprises, the performance of employees and enterprises are both reduced. An employee's shirking of responsibility and moral behavior makes the enterprise incentive mechanism full of risk and liable to stochastic variation (i.e., randomness). However, it is difficult to accurately predict the risk and the randomness so that a rational enterprise can take measures to avoid them. As can be seen from the Figure 2, the ratio of risk allocation of enterprise is $\mathrm{P}_{2}$, and $\mathrm{P}_{2}>\mathrm{P}^{*}$. When the ratio of risk allocation of enterprise is too high the motivation mechanism fails.

When the incentive compensation remains unchanging, and if the randomness increase, the compensation depends on random disturbance factor instead of the level of effort. Thus, the enthusiasm of an employee is reduced, and they will choose a lower optimal work levelq $\mathrm{q}^{*}$. In order to avoid risk and reduce incentive compensation, rational enterprises create a competitive culture to make employees work in a higher-pressure environment. When the enterprise transfers too much risk to employees, and the ratio of risk allocation of employee is high, the turnover rate increases due to the excessive pressure; the culture is then challenged, and the performance of both the employees and the enterprises decrease. As Figure 2 indicates, the ratio of risk allocation of enterprise is $\mathrm{P}_{1}$, and $\mathrm{P}_{1}>\mathrm{P}^{*}$. When the ratio of risk allocation of employees is higher than the ratio of the enterprise, the motivation mechanism fails, but the enterprise will take the responsibility for the loss caused by the imbalance ratio of risk allocation.

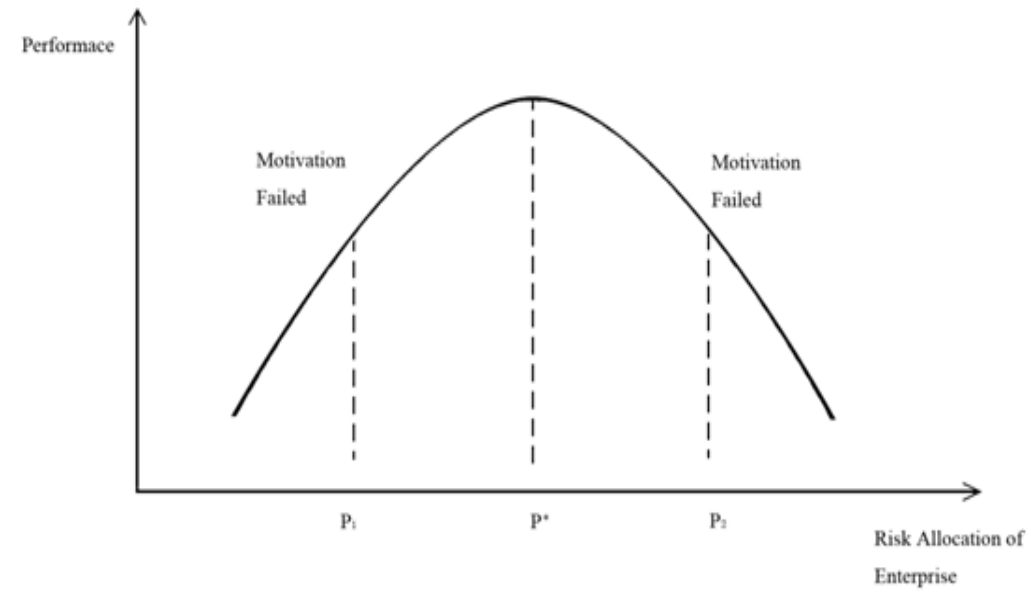

Figure 2. The Optimal Risk Allocation and Performance

As Figure 2 indicates, optimal risk allocation can result in the optimization of enterprise performance. If $\mathrm{P}<\mathrm{P}^{*}$ (such as $\mathrm{P}_{1}$ ), the ratio of risk allocation of employee is lower than enterprises', and the excessive risk transferring leads to motivation mechanism fail. If $\mathrm{P}<\mathrm{P}^{*}$ (such as $\mathrm{P}_{2}$ ), the ratio of risk allocation of enterprise is higher than employee's, and thus insufficient competition also leads to the motivation mechanism fail.

According to the principles of risk allocation, the enterprise will weigh the ratio of risk allocation and the desired level of performance when designing a motivation mechanism; 
enterprises can then adjust the ratio risk allocation constantly. If the ratio of risk allocation of an enterprise is too high, they will transfer part of the risk to employee. If the ratio of risk allocation of enterprise is too low, they will take the initiative to assume part of the risk. Successful enterprise adjust the ratio of risk allocation is in order to find an optimal ratio of risk allocation - both to mobilize the enthusiasm of employee and to maximize the performance.

The reason of risk transferring can be attribute for the pursuit of maximize performance and maximize the benefits, and enterprise is not willing to allocate the high ratio of risk allocation. Unpredictable risk and asymmetric information access are circumstances that are beyond the control of enterprises and employees. Therefore, optimizing the ratio of risk allocation is the most effective solution to solving the problem of a malfunctioning motivation mechanism. Additionally, risk monitoring is necessary, which can reduce the probability of the motivation mechanism failing and can help enterprises to take prompt corrective measures to ensure the employee's behavior does not deviate from the expected target. However, the lack of risk monitoring and supervising employees will also lead to a failure of motivation mechanism. Thus, under the condition of the optimal ratio of risk allocation, enterprises should take risk monitoring and supervising employee into consideration.

The main conclusion is based on the above analysis: Enterprises (principal) and employees (agent) cannot evaluate and monitor risk accurately because of asymmetric information access. An imbalance of ratio of risk allocation is the main circumstance that leads to the failure of a motivation mechanism. Enterprises and employees must adjust the ratio of risk allocation to make it balanced in a dynamic game, and the optimal ratio of risk allocation should meet the maximization of both enterprise and employees. Therefore, the optimal ratio of risk allocation $\mathrm{P}^{*}$ should achieve two goals: 1) Motivate employees effectively and improve their enthusiasm and performance 2.) Motivate enterprise risk monitoring and supervise employees' behavior in order to avoid risk and reduce the probability of failure in the motivation mechanism.

\section{Conclusions and Discussion}

Based on the principal-agent theory and a dynamic game model, this paper analyzes the risk allocation in motivation mechanism, and the conclusions can be obtained:

In the motivation mechanism, if the cost of supervision is larger than the punishment of employees without efforts, risk transferring moderately can motivate employees to work hard. However, risk transferring excessively will make the ratio of risk allocation imbalance and the motivation mechanism fails. In this circumstance, the enterprise should strengthen to supervise employees, punish their moral behavior and to motivate employees allocate risk in order to reduce the probability of failure of the motivation mechanism.

If the cost of employees' effort is less than the benefit of employees' effort, the higher unit cost which employees' effort, the higher income. It shows that the ratio of risk allocation of employees in the motivation mechanism, and it makes employees are enthusiasm in working hard, and the performance will be increased. However, on the contrary, if employees allocate much more risk, the performance will be reduced. Thus, as a principal, the enterprise should take the moderate principle into consideration when the enterprise transfers risk to employees. And the enterprise should adjust the ratio allocation reasonably.

If the cost of employees' effort is more than the income and the punishment combined, the optimal strategy of employees is not work hard. Thus, the motivation mechanism cannot motivate employees to realize the goals when the compensation remains unchanged. In this circumstance, the enterprises motivate employees to work hard by transferring risk to employees and using the competition of culture and market.

In such a circumstance, the failure is not due to the motivation mechanism being incongruent with the benefit of enterprise instead; the failure is due to an imbalanced ratio 
of risk allocation between the principal and agent. Through the above analysis, the paper finds that the best motivation mechanism depends on the optimal ratio of risk allocation between enterprise and employees. There is a complex interactive relationship between risk allocation and risk monitoring in the practice of management, and they promote each other reciprocally in a certain ways. The complexity of management practice is reflected in the optimization problem. This paper asserts that the optimal ratio of risk allocation, which improves towards the optimal in a dynamic game, should satisfy two conditions in order to avoid risk: (1) motivate employees to work efficiently and (2) motivate enterprises to monitor the risk and supervise employees.

This paper suggests the following recommendations for further research and discussion: First, the risk allocation between a principal and an agent should be discussed externally in a dynamic and complex game, extended in subsequent analysis to multi-stage dynamic game, and discussed in relation to the supervision of agent; second, future research should take the dynamic game power into consideration. In reality, a change in dynamic game power will result in a corresponding change in optimal ratio of risk allocation.

\section{References:}

[1]. W.-J. Zhang, and J.-F. Peng, "Motivation System in Chinese Knowledge - Enterprises", Science Research Management, vol. 6, (2001), pp. 18-22.

[2]. A. O. Vega, "Risk Allocation in Infrastructure Financing", Journal of Project Finance, vol. 3, no. 2, (1997), pp. 38-42.

[3]. W.-Y. Zhang, "The relationship of Principal-agent: theoretical analysis and policy implications in the public sector of economy”, Economic Research Journal, vol. 4, (1995), pp. 10-20.

[4]. C. Michael, Jesen, H. William and Mecking, "Theory of the Firm Managerial Behavior, Agency Costs and Ownership Structure", Journal of Financial Economics, vol.3, no. 4, (1975), pp. 305-360.

[5]. W.-Y. Zhang, "Ownership, governance structure and the principal-agent relationship: some comments on Cui Zhiyuan and Zhou Qiren”, Economic Research Journal, vol. 9, (1996), pp. 3-15.

[6]. S.-Y. Xie, "Economic game theory", China Fudan University Press, vol. 1, (2006), pp. 139-155.

[7]. J. A. Mirrlees, "The Optimal Structure of Authority and Incentives Pay in Agency Contracts", Journal of Financial Economics, vol. 7, no. 1, (1976), pp. 105-131.

[8]. D. E. Terpstra and E. J. Rozell, "The Relationship of Staffing Practices to Organizational Lever Measures of Performance", Personnel Psychology, vol. 46, no. 1, (1993), pp. 27-48.

[9]. Akintoye, C. Taylor and E. Fitzgerald, "Risk Analysis and Management of Private Finance Initiative Projects", Engineering, Construction and Architectural Management, vol. 5, no.1, (1998), pp. 9-21.

[10].X. Qi, Y.-J. Ke and S.-Q. Wang, "Analysis on critical risk factors causing the failure of China's PPP project”, China soft science magazine, vol. 5, (2009), pp. 751-755.

[11].K. C. Lam and W. S. Chow, "The Significance of Financial Risks in BOT Procurement. Building Research and Information, vol. 27, no. 2, (1999), pp. 84-95.

[12].B. Li, Akintoye, P. J. Edwards and C. Hardcastle, "The Allocation of Risk in PPP/PFI Construction Projects in the UK", International Journal of Project Management, vol. 23, no. 1, (2005), pp. 25-35.

[13].J.-B. Song, D.-R. Song and J. Shan, "Study on risk allocation in BOT project of refuse-incineration power generation", China soft science magazine, vol.7, (2010), pp. 71-79.

[14].L.-Y. Shen, A. Platten and X. P. Deng, "Role of the public private partnerships to manage risks in public sector projects in Hong Kong”, International Journal of Project Management, vol. 24, no. 7, (2004), pp. 587-594.

[15].W.-B. Duan and S. Yuan, "Risk allocation and incentive contracts: a further review of the principal-agent theory", Nankai Economic Studies, vol. 5, (2004), pp. 19-25.

[16].S. J. Grossman and O. Hart, "The cost and benefits of ownership: A theory of vertical and lateral integration", Journal of Political Economy, vol. 94, no. 4, (1986), pp. 691-719.

[17].O. Hart and J. Moore, "Property Rights and the Nature of the Firm", Journal of Political Economy, vol. 98, no. 6, (1990), pp. 1119-1158.

[18]. M. E. Porter and C.-V.-D. Linde, "Green and competitive: Ending the stalemate", Harvard Business Review, vol. 73, no. 5, (1995), pp. 120-134.

[19].T. Guimaraes and K. Liska, "Exploring the business benefits of environmental stewardship", Business Strategy and the Environment, vol. 4, no. 1, (1995), pp. 9-22.

[20].Q.-H. Zhu and Y.-J. Dou, "Exploring the business benefits of environmental stewardship in Green Supply Chains", System Engineering - Theory \& Practice, vol. 27, no. 12, (2008), pp. 85-89. 